\title{
Small Peptides against the Mutant SOD1/Bcl-2 Toxic Mitochondrial Complex Restore Mitochondrial Function and Cell Viability in Mutant SOD1-Mediated ALS
}

\author{
Wenzhi Tan (谭文之), ${ }^{\star}$ Nicole Naniche,* Alexey Bogush, Steve Pedrini, Davide Trotti, and Piera Pasinelli \\ Frances and Joseph Weinberg Unit for ALS Research, Farber Institute for Neurosciences, Department of Neuroscience, Thomas Jefferson University, \\ Philadelphia, Pennsylvania 19107
}

\begin{abstract}
Mutations in superoxide dismutase 1 (SOD1) cause amyotrophic lateral sclerosis (ALS) in $20 \%$ of familial cases (fALS). Mitochondria are one of the targets of mutant SOD1 (mutSOD1) toxicity. We previously demonstrated that at the mitochondria, mutSOD1 forms a toxic complex with Bcl-2, which is then converted into a toxic protein via a structural rearrangement that exposes its toxic BH3 domain (Pedrini et al., 2010). Here we now show that formation of this toxic complex with Bcl-2 is the primary event in mutSOD1-induced mitochondrial dysfunction, inhibiting mitochondrial permeability to ADP and inducing mitochondrial hyperpolarization. In mutSOD1-G93A cells and mice, the newly exposed $\mathrm{BH} 3$ domain in $\mathrm{Bcl}-2$ alters the normal interaction between $\mathrm{Bcl}-2$ and $\mathrm{VDACl}$ thus reducing permeability of the outer mitochondrial membrane. In motor neuronal cells, the mutSOD1/Bcl-2 complex causes mitochondrial hyperpolarization leading to cell loss. Small SOD1-like therapeutic peptides that specifically block formation of the mutSOD1/Bcl-2 complex, recover both aspects of mitochondrial dysfunction: they prevent mitochondrial hyperpolarization and cell loss as well as restore ADP permeability in mitochondria of symptomatic mutSOD1-G93A mice.
\end{abstract}

\section{Introduction}

Amyotrophic lateral sclerosis (ALS) is an adult-onset, neurodegenerative disorder characterized by loss of motor neurons (Pasinelli and Brown, 2006). Approximately 2\% of ALS arises from mutations in $\mathrm{Cu} / \mathrm{Zn}$ superoxide dismutase (SOD1) (Rosen et al., 1993), which acquires a new, yet unidentified, toxic function (Pasinelli and Brown, 2006).

Mitochondria are a target of disease in ALS and a specific target of toxicity of mutant SOD1 (mutSOD1). Normally a cytosolic protein, $\sim 1-2 \%$ of SOD1 is found at the mitochondria (Higgins et al., 2002; Mattiazzi et al., 2002; Liu et al., 2004; Vande Velde et al., 2008) where, when mutated, it forms high molecular weight insoluble aggregates (Pasinelli et al., 2004) and it interacts with, and alters the function of, several proteins of the outer mitochondrial membrane (OMM) (Israelson et al., 2010; Pedrini et al., 2010). Ultimately, multiple mitochondrial functions are impaired from calcium buffering capacity (Damiano et al., 2006),

\footnotetext{
Received Nov. 20, 2012; revised May 15, 2013; accepted June 4, 2013.

Author contributions: W.T., N.N., D.T., and P.P. designed research; W.T. and N.N. performed research; A.B. and S.P. contributed unpublished reagents/analytic tools; W.T., N.N., and P.P. analyzed data; W.T., N.N., D.T., and P.P. wrote the paper.

This work was supported by the National Institutes of Health (Grants R01-NS051488 to P.P. and R01-NS064488 to D.T.) and by the Farber Family foundation. We thank Mr. Michael Goffredo for maintaining the mouse colony and providing the mice used in this work (control and mutSOD1-G93A)

*W.T. and N.N. contributed equally to this work.

The authors declare no competing financial interests.

Correspondence should be addressed to Dr. Piera Pasinelli, Weinberg Unit for ALS Research, Farber Institute for Neurosciences and Department of Neuroscience, Thomas Jefferson University, 900 Walnut Street, JHN-451, Philadelphia, PA 19107. E-mail: piera.pasinelli@jefferson.edu.

DOI:10.1523/JNEUROSCI.5385-12.2013

Copyright $\odot 2013$ the authors $\quad 0270-6474 / 13 / 3311588-11 \$ 15.00 / 0$
}

to electron transport (Mattiazzi et al., 2002; Jung et al., 2002; Kirkinezos et al., 2005) to morphology (Kong and Xu, 1998). To develop drugs with broad effects on multiple mitochondrial functions we need to define the order of events leading to mitochondrial demise, and define and how and if mutSOD1 impairs each function.

To design potent compounds that target the key mediators of mutSOD1 toxicity, we dissected the relationship between mutSOD1 and its proposed mitochondrial partners, Bcl-2 and VDAC1 in particular, and analyzed the order of events downstream of mutSOD1 that destabilize the mitochondria. In the OMM, mutSOD1 aberrantly binds to and forms a toxic complex with mitochondrial Bcl-2, which is converted into a toxic protein via a conformational modification that exposes its otherwise hidden BH3 domain (Pedrini et al., 2010). Misfolded mutSOD1 also binds VDAC1 in spinal cord mitochondria (Israelson et al., 2010). However, Bcl-2 is also a known binding partner and regulator of VDAC1 in the OMM (Arbel and Shoshan-Barmatz, 2010). Since mutSOD1 binds spinal cord mitochondria in the absence of VDAC1 (Li et al., 2010) perhaps in ALS, misfolded mutSOD1 targets VDAC1 not directly, but through its aberrant interaction with Bcl-2 and the resulting impaired VDAC1 function is only one of the aspects of mitochondrial dysfunction in ALS.

Here we demonstrate that $\mathrm{Bcl}-2$, and not VDAC1, is the major mediator of mutSOD1 toxicity at the mitochondria and that the mutSOD1/Bcl-2 complex acts upstream of VDAC1 to reduce mitochondrial ADP permeability and, in parallel but perhaps independently of VDAC1, induces hyperpolarization of the OMM. We show that SOD1 and VDAC1 independently bind to, and 
compete for, Bcl-2. The aberrant binding to mutSOD1 changes $\mathrm{Bcl}-2$ conformation altering its normal binding to $\mathrm{VDAC1}$; this reduces mitochondrial ADP permeability. In the mutSOD1G93A mice the binding between conformationally changed Bcl-2 and VDAC1 becomes stronger as disease progresses and this is paralleled by a progressive decrease in mitochondrial ADP permeability. The mutSOD1/Bcl-2 complex also triggers hyperpolarization of the OMM leading to cell loss. SOD1-like peptides that block formation of the mutSOD1/Bcl-2 complex without affecting directly VDAC1, block hyperpolarization, prevent cell death, and restore mitochondrial ADP permeability in symptomatic mutSOD1-G93A mice. Thus, by restoring two key, and probably not sequentially related, aspects of mitochondrial dysfunction these peptides against the mutSOD1/ Bcl-2 complex offer the opportunity for broad spectrum protection of mitochondrial function offering better efficacy in affecting disease progression.

\section{Materials and Methods}

Cell culture, transfections, viral transduction, peptide delivery, and viability assay. HEK293T and NSC-34 cells were maintained in DMEM, 10\% (v/v) fetal bovine serum (FBS) and pen/strep. Stably transfected control and VDAC1 knockdown cells were maintained under puromycin and removed before experiments. Cells were either transfected with Lipofectamine 2000 (Invitrogen) for $48 \mathrm{~h}$ or transduced with adenovirus harboring SOD1 genes for $72 \mathrm{~h}$ at a multiplicity of infection of 20. Peptides were administered via Chariot (Active Motif) for $2 \mathrm{~h}$ before assay. Cell viability was scored using the CellTiter-Glo Luminescence Viability Assay (Promega). All follow manufacturer's protocols.

Isolation of mitochondria. Percoll purification for ADP consumption assay: Mitochondria from mice spinal cords were isolated according to the method of Sims and Anderson (2008) at $4^{\circ} \mathrm{C}$. Crude pellet isolation for coimmunoprecipitation (co-IP) experiments: for disease course co-IP and Western blots, spinal cord mitochondria were isolated following the protocol from Pallotti and Lenaz (2007). Isolation of mitochondria from HEK293T cells: mitochondria from cell cultures were isolated according to Parsons et al. (1966) except that cells were broken with nitrogen cavitation protocol of Kristián et al. (2006); isolations were performed at $4^{\circ} \mathrm{C}$.

Animals. Nontransgenic and transgenic mutSOD1-G93A ALS (The Jackson Laboratory) mice were maintained on a C57J/B6 background. One to three spinal cords of both male and female mice were used per condition of experiment. All animal procedures were approved by the Animal Care and Use Committee of Thomas Jefferson University.

Electron microscopy of mitochondrial morphology. Samples were fixed in $2 \%$ glutaraldehyde with $1 \%$ tannic acid buffered in phosphate buffer, $\mathrm{pH} 7.4$, for overnight at $5^{\circ} \mathrm{C}$, rinsed in the same buffer, and then exposed to $2 \%$ osmium tetroxide. Following a rinse with double distilled water, samples were put in $0.5 \%$ uranyl acetate and then dehydrated in graded steps of acetone (one wash at 25, 50, 75, and 95\% and three washes at $100 \%)$. Samples were then infiltrated with Spurr's resin and polymerized in a $65^{\circ} \mathrm{C}$ convection oven. The blocks were thin sectioned $(70 \mathrm{~nm})$ with a Diatome diamond knife on a Leica UCT ultramicrotome, sections were analyzed in an FEI Tecnai $12 \mathrm{~T}$ electron microscope, and images were digitally recorded with an AMT XR111 camera.

Generation of SOD1 deletion mutants. Recombinant DNAs were created within the pcDNA3.1 $(+)$ backbone. Enhanced green fluorescent protein (EGFP) control plasmid was created by PCR of pEGFP-C1 (Clontech) with the following two primers: cgcgaattcgccaccatggtgagcaagggcgaggag, cgcctcgagttacttgtacagctcgtcca and then inserted into EcoRI and XhoI sites within pcDNA3.1(+). EGFP plasmid without Kozak sequence (pEGFP) was created using a similar method with another two primers: cgcgaattcatggtgagcaagggcgaggag, cgcctcgagttacttgtacagctcgtcca. This later plasmid pEGFP was used to create SOD1 mapping plasmids by PCR of SOD1 cDNA with combinations of primers corresponding to SOD1 sequences of various exons and inserted into BamHI and XhoI sites within pEGFP.
Measurement of OMM permeability to ADP. A portion of the mitochondrial suspension $(\sim 15 \mu \mathrm{g}$ of mitochondrial protein) was diluted into $50 \mu \mathrm{l}$ of respiration buffer containing $5 \mathrm{~mm}$ succinate, $0.3 \mathrm{M}$ mannitol, $10 \mathrm{~mm} \mathrm{NaH} \mathrm{PO}_{4}, 5 \mathrm{~mm} \mathrm{MgCl}_{2}$, and $10 \mathrm{~mm} \mathrm{KCl,} \mathrm{pH} \mathrm{7.2.} \mathrm{The} \mathrm{mito-}$ chondrial respiration was measured after each addition of $100 \mu \mathrm{M}$ ADP according to the method of Lee et al. (1994). AMP (50 $\mu \mathrm{M})$ was also added to inhibit adenylate kinase activity (Szasz et al., 1976). Only the transition between state III and IV respiration was shown to illustrate the membrane permeability per maximum ADP stimulated respiration activity. The oxygen consumption was measured by using a Clark oxygen electrode after addition of $5 \mathrm{~mm}$ succinate and $100 \mu \mathrm{M}$ ADP. The result was converted to ADP consumption by $\mathrm{P} / \mathrm{O}$ ratio (two for succinate as the substrate). The membrane permeability to ADP was obtained by fitting the following equations:

$$
\text { Flux }=\frac{d[A D P]}{d t}=-P * A *\left(C_{o}-C_{i}\right)=-\frac{V_{\max } * C_{i}}{K_{m}+C_{i}} d t
$$

$$
C_{o}^{t+d t}=C_{o}^{t}+\frac{d[A D P]}{d t} d t=C_{o}^{t}-\frac{V_{\max } * C_{i}}{K_{m}+C_{i}} d t
$$

where $P$ is the permeability of the OMM to ADP; $A$ is the total area; and $V_{\max }$ and $K_{\mathrm{m}}$ are the enzyme kinetics of adenine nucleotide translocase. $V_{\max }$ can be calculated from the maximum ADP-simulated respiration, which also suggests the relative amount of coupled mitochondria. $K_{\mathrm{m}}$ is taken as $4.3 \mu \mathrm{M} . C_{i}$ and $C_{o}$ are the ADP concentrations in the intermembrane space and medium, respectively, whose relationship can be calculated from first equation. $P^{\star} A$ was allowed to vary to achieve the best fit of medium $[\mathrm{ADP}]$ as a function of time.

Immunofluorescence. Fixed cells: Cells were fixed with $2 \%$ paraformaldehyde/ $2 \%$ sucrose, permeabilized by ice-cold methanol, blocked by $5 \%$ FBS, incubated with rabbit anti-Bcl-2 antibody (1:200; Millipore) and then secondary anti-rabbit Alexa Fluor 405 (1:200; Invitrogen), followed by analysis of confocal microscopy. Confocal imaging was done with an Olympus confocal microscope. For MitoTracker staining, cells were stained with $300 \mathrm{~nm}$ deep red MitoTracker (Invitrogen) and incubated at $37^{\circ} \mathrm{C}$ for $30 \mathrm{~min}$ before fixation. Cells were blocked in $2 \% \mathrm{FBS}$ for $20 \mathrm{~min}$, then treated with $25 \mathrm{nM}$ tetramethylrhodamine ethyl ester (TMRE; Abcam) for $20 \mathrm{~min}$. Following treatment, cells were washed $2 \times$ with DMEM, then covered with HEPES-buffered DMEM without phenol red or FBS. Slides were then imaged using Zeiss microscopy.

Western blots and co-IPs. The following antibodies were used for Western blots: horseradish peroxidase (HRP)-conjugated anti-VDAC1 (1: 5000; Santa Cruz Biotechnology), VDAC2 (1:500, goat; Abcam), CoxIV (1:1000, rabbit; Cell Signaling Technology), HRP-conjugated anti-Bcl-2 (1:1000; Santa Cruz Biotechnology), SOD1 (1:1000, sheep; Calbiochem), GAPDH (1:10,000, mouse; Abcam), and GFP (1:1000, mouse; Clontech). Secondary antibodies were used at a 1:5000 dilution, and were either from Jackson ImmunoResearch (anti-sheep and goat) or from GE Healthcare (anti mouse and rabbit). For co-IP $250 \mu \mathrm{g}$ of crude mitochondria or total cell lysates precleared with $20 \mu \mathrm{l}$ of Protein A beads (Qiagen) were incubated with appropriate antibodies, and pulled down with $20 \mu \mathrm{l}$ of Protein A beads. Antibodies for co-IPs were the following: anti-Bcl-2 (rabbit; Calbiochem), anti-pocket-Bcl-2 (mouse; BD Biosciences), anti-BH3-Bcl-2 (rabbit; Calbiochem), and rabbit IgG (Santa Cruz Biotechnology), anti-Bcl-2 (rabbit; Millipore), anti-GFP (rabbit; Clontech), and anti-VDAC (rabbit; Santa Cruz Biotechnology). In peptide-blocking experiments, $1 \mu \mathrm{m}$ peptides were added to the IP buffer. All Western blots quantified using Quantity One software to analyze images taken on a ChemiDoc.

Statistical analyses. ANOVA followed by Fisher post hoc was used for multiple comparisons. Student's two-tailed, unpaired $t$ test was used to compare two groups, unless otherwise specified. Data are represented as means \pm SEM. 


\section{Results}

Bcl-2 strongly interacts with both SOD1 and VDAC1 and mediates

their interaction

At the mitochondria, mutant but not wild-type (WT) SOD1, has been shown to bind VDAC1 and, based on in vitro experiments done in reconstituted lipid bilayer, it has been proposed that mutSOD1 alters mitochondrial ionic homeostasis by closing VDAC1 (Israelson et al., 2010). Whether mutSOD1 directly binds and impairs VDAC1 in spinal cord mitochondria is still not clear. While the lipid bilayer experiments-where mutSOD1 alone inhibited VDAC1 conductancewould argue in favor of a direct effect, these experiments are highly artificial (a setting where only two proteins are expressed in an artificial system that lacks the important regulatory elements otherwise present in the OMM); other evidence would suggest otherwise. Indeed, it has been reported that mutSOD1 can still bind spinal cord mitochondria in the absence of VDAC1 (Li et al., 2010). In light of the evidence that $\mathrm{Bcl}-2$ binds VDAC1 and regulates its gating properties (Arbel and Shoshan-Barmatz, 2010) and based on our findings that in mouse and human spinal cord mitochondria, mutSOD1 aberrantly binds $\mathrm{Bcl}-2$ and changes its conformation, we hypothesized that rather than directly the mutSOD1 affects VDAC1 function through a conformationally changed Bcl-2. Thus, to elucidate the binding relationships between mutSOD1, Bcl-2, and VDAC1, we first transfected HEK293T cells that do not express detectable levels of Bcl-2 (Pedrini et al., 2010) with combinations of SOD1, $\mathrm{Bcl}-2$, and VDAC1; i.e., Bcl-2 and SOD1 \pm VDAC1, Bcl-2 and VDAC1 \pm SOD1, VDAC1, and SOD1 \pm Bcl-2. We found that SOD1 (WT or mutant) binds to Bcl-2 equally in the presence or absence of VDAC1 (Fig. 1A), although mutSOD1G93A has increased binding affinity for Bcl-2 compared with SOD1-WT, as we have previously noted (Pasinelli et al., 2004; Pedrini et al., 2010). Similarly, VDAC1 binds to Bcl-2 equally well in the presence or absence of SOD1, either WT or G93A (Fig. 1B). These results demonstrate a direct binding between (1) SOD1 and Bcl-2 and (2) Bcl-2 and VDAC1, both of which have been previously demonstrated (Pedrini et al., 2010; Arbel and Shoshan-Barmatz, 2010). However, in the presence of Bcl-2, the apparent direct binding between SOD1 and VDAC1 practically disappears (Fig. 1C). To confirm this, we also performed co-IP between mutSOD1 and VDAC1 with increasing Bcl-2 levels. As a result, the amount of mutSOD1 pulled down together with VDAC1 decreases as the amount of Bcl-2 increases (Fig. $1 E)$, further indicating that SOD1 and VDAC1 compete for the

$\mathrm{Bcl}-2 / \mathrm{SOD} 1$

transfection ratio
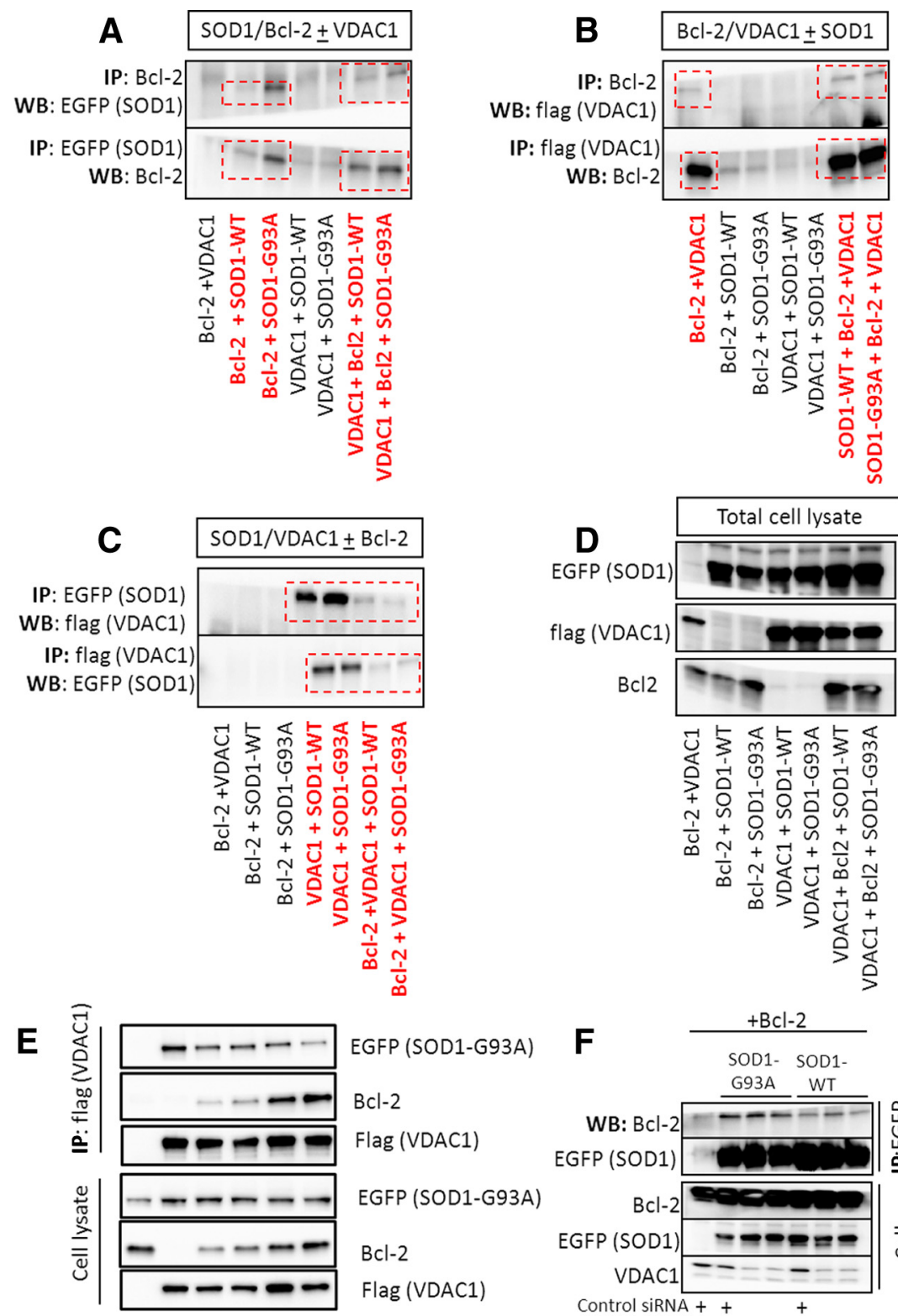

Figure 1. $\mathrm{BCl}-2$ mediates the binding between SOD1 and VDAC1. $A-D, B C l-2, E G F P$-tagged SOD1 (WT or G93A), and flag-tagged VDAC1 were transfected into HEK293T (SOD1 and VDAC1 \pm BCl-2; VDAC1 and BCl-2 \pm SOD1; BCl-2 and VDAC1 \pm S0D1). Cells were harvested $24 \mathrm{~h}$ later and cell lysates were immunoprecipitated with the indicated antibodies $(\boldsymbol{A}-\boldsymbol{C})$ and probed for individual proteins. D, WB of total lysates for protein expression. $\boldsymbol{E}, \mathrm{BCl}$-2 and EGFP-tagged mutSOD1-G93A were cotransfected into HEK293T at the indicated ratio, together with a constant amount of flag-tagged VDAC1. Cells were harvested $24 \mathrm{~h}$ later and cell lysates were immunoprecipitated with flag antibody and probed for individual proteins. $\boldsymbol{F}$, HEK293T cells were knocked down with either control or VDAC1 siRNAs. (ells were then transfected with BCl-2 and EGFP-tagged S0D1 (WT or G93A), lysed, immunoprecipitated, and probed with indicated antibodies.

binding with $\mathrm{Bcl}-2$ and suggesting that, at least in an overexpression system, Bcl-2 mediates the interaction between mitochondrial SOD1 and VDAC1.

To rule out the possibility that endogenous VDAC1 may mediate the interaction between SOD1 and Bcl-2, we knocked down VDAC1 with two independent siRNAs in HEK293T cells and performed cross co-IP experiments as above. Western blot analysis confirmed that $>80 \%$ of VDAC1 was knocked down (Fig. $1 F)$. Co-IP results show that the binding between Bcl-2 with SOD1 (WT or G93A) is not affected by VDAC1 knockdown, further indicating that SOD1 interacts with Bcl-2 independently 

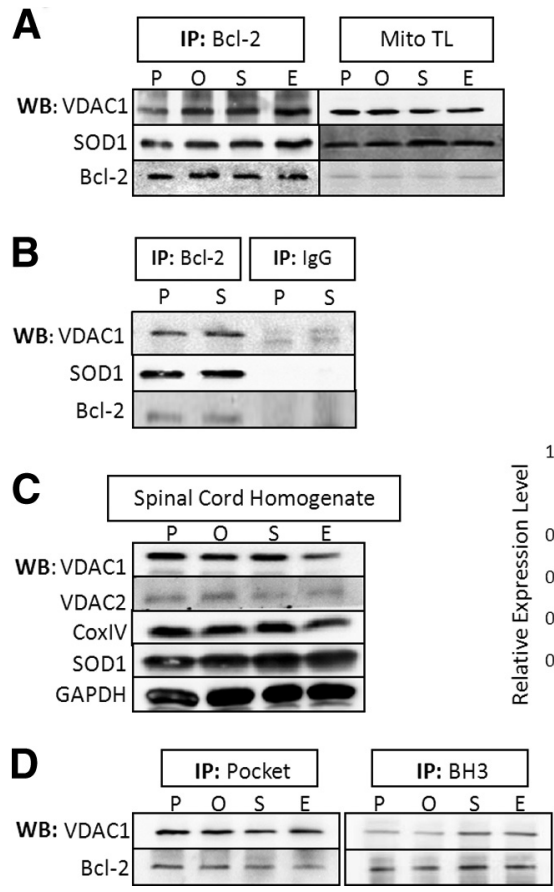

Figure 2. BH3 domain exposed $\mathrm{BCl}-2$ binds to VDAC1 with increased affinity through the course of disease. $A$, Crude mitochondria isolated from mutSOD1-G93A mice at indicated disease stages (P, presymptomatic; 0 , onset; $S$, symptomatic; $E$, end stage) were lysed and immunoprecipitated with anti-Bcl-2 antibody and then probed with indicated antibodies. Quantifications of co-IPs were normalized to pulldown of BCl-2. B, Control IPs with either anti-BCl-2 or IgGs. C, Western blot (WB) to assess total amount of VDAC1, VDAC2, COXIV, and SOD1 throughout disease progression. GAPDH was also measured as a control at each disease stage. Quantifications of WBs were normalized to each protein level at presymptomatic stage $\left(n=3,{ }^{*} p \leq 0.05\right)$. D, BCl-2 was immunoprecipitated using indicated conformational specific antibodies and amount of VDAC1 co-IP was assessed over disease progression.

of VDAC1. Altogether, these results suggest VDAC1 as a downstream target of the SOD1/Bcl-2 complex, and indicate that Bcl-2 acts as a bridge between SOD1 and VDAC1.

\section{Binding between conformationally modified Bcl-2 and VDAC1 increases over disease progression in mutSOD1-G93A mice}

We next studied the relationship between the three proteins in vivo. We isolated spinal cord mitochondria from mutSOD1G93A transgenic mice at different stages of disease and used anti Bcl-2 antibodies to co-IP VDAC1 and SOD1, respectively (Fig. 2 ). As expected, both VDAC1 and SOD1 co-IP with Bcl-2. Interestingly, however, we found that the binding between VDAC1 and $\mathrm{Bcl}-2$ increases as disease progresses (Fig. $2 \mathrm{~A}$ ). In fact, the amount of VDAC1 pulled down by the Bcl-2 antibody increases throughout disease despite a decrease in overall VDAC1 expression levels (Fig. $2 A, C$ ). Concomitant with a decrease in VDAC1 expression, we found that levels of VDAC2 and CoxIV are also significantly reduced over the course of the disease (Fig. 2C), in agreement with previous reports demonstrating decreased expression of various mitochondrial proteins in mutSOD1 versus SOD1-WT mouse models (Li et al., 2010). Bcl-2 also co-IPs with mutSOD1 as we have shown previously (Pedrini et al., 2010); although contrary to what we observed for VDAC1, the amount of monomeric SOD1 pulled down by the anti-Bcl-2 antibody is consistent throughout disease. Additionally, expression levels of mutSOD1 do not change throughout disease progression. Control IgGs immunoprecipitated neither VDAC1 nor SOD1, proving the specificity of both co-IP experiments (Fig. 2B). Thus, also in spinal cord mitochondria Bcl-2 mediates the interactions between mutSOD1 and the OMM and eventually VDAC1.

In ALS mice and patients, Bcl-2 undergoes a progressive conformational change triggered by the aberrant interaction with mutSOD1, which exposes its toxic BH3 domain (Pedrini et al., 2010). To determine whether this mutSOD1-induced conformational modification in $\mathrm{Bcl}-2$ accounts for the increased interaction between $\mathrm{Bcl}-2$ and VDAC1 through disease progression, we again isolated spinal cord mitochondria from mutSOD1-G93A mice at different stages of disease and performed co-IP with two different conformation-specific anti$\mathrm{Bcl}-2$ antibodies as previously reported (Pedrini et al., 2010). Specifically, we used an anti-pocket-Bcl-2 antibody (which recognizes the normally structured and functional Bcl-2) or an anti-BH3 antibody (which should not recognize and pull down Bcl-2 unless it underwent a conformational change with $\mathrm{BH} 3$ domain exposure). Interestingly, in symptomatic and end-stage mutSOD1-G93A mice, the anti-BH3 antibody co-IPs VDAC1 (Fig. 2D) and the amount of VDAC1 pulled down by the anti-BH3 antibody increases as disease progresses, while it decreases with the anti-pocket antibody (Fig. 2D). This pattern of co-IP strongly correlates with our previous observation that as disease progresses, Bcl-2 gradually exposes the toxic $\mathrm{BH} 3$ domain and reduces the pocket domain. These data strongly indicate that it is the disease-driven conformational change in Bcl-2 that accounts for the increase in binding between Bcl-2 and VDAC1 over disease progression in mice. They also confirm that Bcl-2 mediates the toxic interaction of mutSOD1 with the mitochondria in vivo.

\section{The mutSOD1/Bcl-2 toxic complex decreases mitochondrial} ADP permeability and impairs cellular viability

To confirm that formation of the mutSOD1/Bcl-2 complex, and the subsequent exposure of the toxic $\mathrm{BH} 3$ domain, is the relevant mechanism in mutSOD1-mediated mitochondrial toxicity, which eventually affects OMM permeability upstream of VDAC1, we performed the following sets of experiments. First, we cotransfected SOD1 and Bcl-2 into HEK293T cells and then isolated mitochondria to perform ADP permeability assays. Since the HEK293T cells do not express detectable levels of Bcl-2 (Pedrini et al., 2010) while having robust expression of VDAC1 (Fig. $3 E$, insert), they are a suitable system to study whether the effects of mutSOD1 on mitochondrial bioenergetics (and other mitochondrial proteins) depend on Bcl-2. Indeed, using this system we showed that the mutSOD1 requires Bcl-2 to alter mitochondrial morphology (Pedrini et al., 2010). For ADP permeability, mitochondria were added into phosphate buffer containing succinate as substrate. Oxygen consumption was recorded after each addition of $100 \mu \mathrm{M}$ ADP and converted into ADP consumption curve as previously published (Lee et al., 1994). Only the transition between state III and state IV respiration was shown to better illustrate the results on ADP permeability. Compared with just 
mutSOD1-G93A transfection or control cells, cotransfection of mutSOD1-G93A and Bcl-2 inhibits mitochondrial ADP consumption at low ADP concentration by $30-$ 40\% (Fig. 3B, insert), indicating a lower permeability to ADP, consistent with reduced VDAC1 gating (Lee et al., 1994). This inhibition is not observed in mitochondria isolated from cells cotransfected with SOD1-WT and Bcl-2 (Fig. 3A), suggesting that the drop in mitochondrial ADP permeability is induced by the mutSOD1/Bcl-2 complex and that, functionally, mutSOD1 requires $\mathrm{Bcl}-2$ to influence $\mathrm{VDAC1}$ gating and therefore mitochondria ADP permeability. The mutSOD1/Bcl-2 complex acts via the toxic $\mathrm{BH} 3$ domain, because cotransfection with mutSOD1-G93A and a $\mathrm{BH} 3$-inactivated mutant form of Bcl-2 (Bcl2-AAA), which is generated by mutating three amino acids at the core of the $\mathrm{BH} 3$ domain (GDD $\rightarrow$ AAA) (Cheng et al., 1997) and abolishes mutSOD1 mitochondrial toxicity (Pedrini et al., 2010), fully rescues the reduction in ADP consumption (Fig. $3 B$, green line). Together with the findings that conformationally modified Bcl-2 increases its binding affinity for VDAC1, these results prove that also functionally VDAC1 is a downstream target of the mutSOD1/ Bcl-2 toxic complex and that mitochondrial mutSOD1 requires a modified Bcl-2 (with the toxic $\mathrm{BH} 3$ domain exposed) to disrupt mitochondrial ADP permeability. To confirm that the mutSOD $1 / \mathrm{Bcl}-2$ complex induces a decrease in ADP consumption primarily targeting the OMM upstream of VDAC1, we also used G3139, an oligonucleotide inhibitor of the VDAC channels (Tan et al., 2007). There is essentially no more inhibition on ADP consumption in mitochondria with mutSOD1-G93A and Bcl-2 in the presence of G3139 (Fig. $3 C)$. Thus, the effects of the mutSOD $1 / \mathrm{Bcl}-2$ complex on mitochondria ADP permeability are mostly mediated through VDAC in the outer membrane.

The mutSOD1/Bcl-2 complex-initiated mechanism of inhibition of mitochondrial ADP permeability also affects cell viability. In the second set of experiments, we generated control and VDAC1 stable knockdown HEK293T cell lines using control shRNA, and VDAC1 shRNA, respectively. Western blot analysis shows $\sim 80 \%$ VDAC1 knockdown in these cells (Fig. 3E, insert). Knocking down of VDAC1 affects neither overall cell viability nor mitochondrial morphology or integrity as measured by MitoTracker and cytochrome $c$ staining (data not shown). We then cotransfected both control knockdown cells (VDAC1 positive) and VDAC1 knockdown cells with either SOD1 (WT or G93A) and Bcl-2 (WT or AAA). Cotransfection of mutSOD1-G93A and Bcl-2 in control (VDAC1 positive), but not VDAC1 knockdown cells, causes a significant $20 \%$ reduction of cell viability $(p \leq 0.05)$, while inactivation of the toxic $\mathrm{BH} 3$ domain in $\mathrm{Bcl}-2$ equally abolishes toxicity of the mutSOD1/Bcl-2 complex in both VDAC1-positive and

E

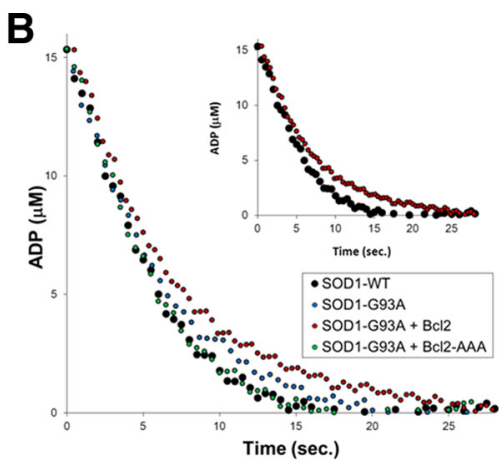

D
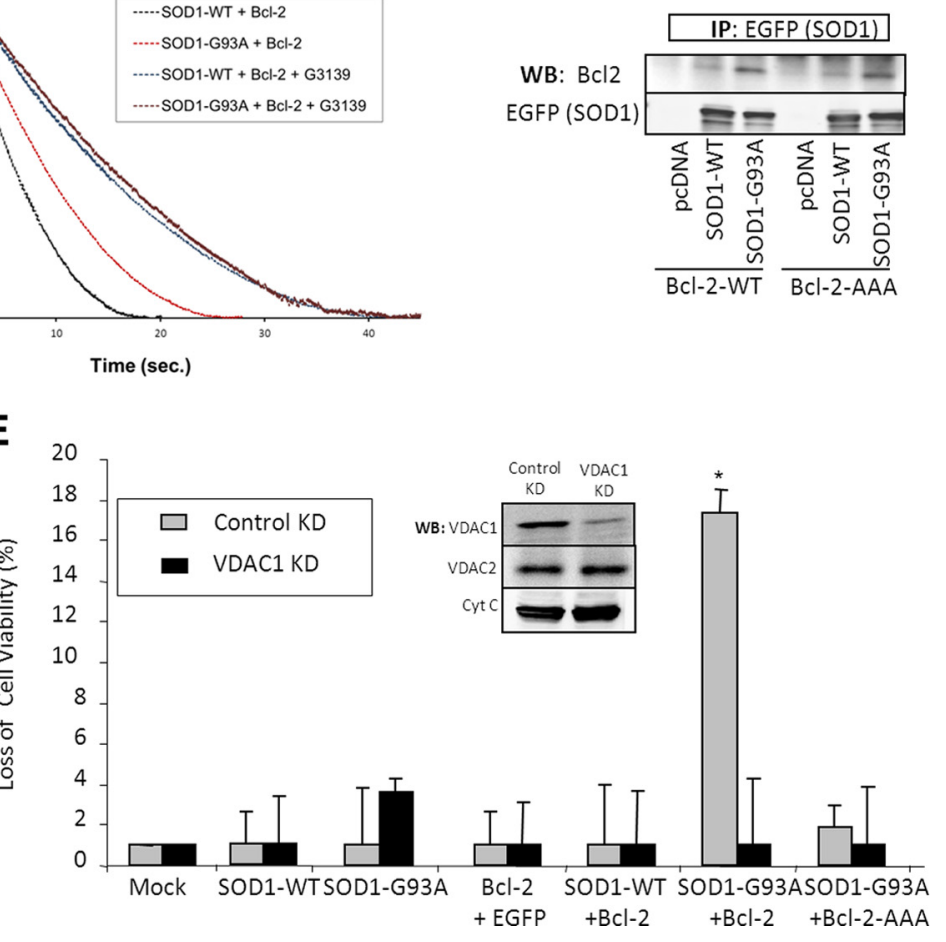

Figure 3. MutSOD1/BCl-2 complex induces decreased mitochondrial ADP permeability and loss of cellular viability. A-C, ADP consumption curves of mitochondria isolated from HEK293T cells cotransfected with pcDNA3, SOD1-WT $\pm \mathrm{BCl}-2(\boldsymbol{A})$ or SOD1-WT mutSOD1-G93A $\pm \mathrm{BCl}-2$ (WT or AAA) (with pcDNA3 and mutSOD1-G93A $\pm \mathrm{BCl}-2$ results highlighted in the inset) (B) or SOD1fected with SOD1 (WT or G93A, fused with EGFP) into HEK293T cells. Lysates were immunoprecipitated with anti-GFP antibody and probed with either anti-GFP or anti-BCl-2 antibody. $\boldsymbol{E}$, Control or VDAC1 stable knockdown (VDAC1 KD) HEK293T cells were transfected with SOD1 (WT or G93A) and pcDNA, BCl-2 (WT or AAA). Cell viability was then measured ( ${ }^{*} p \leq 0.05$ ). Insert, WB of VDAC1, VDAC 2, and cytochrome c (as a mitochondrial loading control) in both cell lines.

knockdown cells (Fig. 3E), confirming that the mutSOD1/Bcl-2 complex acts upstream of VDAC1 and indicating that the trigger event is the formation of the mutSOD1/Bcl-2 complex.

The mutSOD1/Bcl-2 complex becomes a therapeutic target at the mitochondria: generation of small peptides to block mutSOD1 binding to $\mathrm{Bcl}-2$

Based on the knowledge that the mutSOD1/Bcl-2 complex is the initiating event, we then designed a series of small peptides to prevent the formation of this complex. The goal is to use these peptides therapeutically to improve mitochondrial function and bioenergetics, ultimately preventing mutSOD1-mediated motor neuron loss. In general, peptides that block protein-protein interactions can be designed to mimic different domains in either one of the binding partners' proteins. Some strategies, such as in 
cancer therapy, have focused on the design of small molecules that mimic the action of members of the Bcl-2 family and their binding partners, resulting in wide availability of Bcl-2-like peptides.

In view of the critical importance of $\mathrm{BH} 3$ domain(s) in regulating the interaction between $\mathrm{Bcl}-2$ and its partner proteins, BH3-like peptides have mainly been made (Schimmer et al., 2001). However, for our studies we decided to design SOD1-like rather than Bcl-2 (and/or BH3)-like peptides. This is because (1) protein-protein interaction plays a fundamental role in the function of $\mathrm{Bcl}-2$. In addition to inhibiting the binding between mutSOD1 and Bcl-2, Bcl-2-like peptides might inhibit the binding between Bcl-2 and other proteins and, therefore, it might not be clear how any effect (positive or negative) on mitochondrial function and/or cell survival can be uniquely attributed to the lack of interaction between Bcl-2 and mutSOD1. In the long run, given the importance of $\mathrm{Bcl}-2$ for mitochondrial bioenergetics and cell survival, any nonspecific effect of the Bcl-2-like peptides (e.g., blocking binding with pro-apoptotic proteins or altering the binding with ion channels of the OMM) may be detrimental rather than beneficial. We believe that focusing on SOD1 is safer and should provide a more efficacious and specific approach as, to our knowledge, SOD1 does not seem to bind to many other partners and in general, protein-protein interaction does not regulate SOD1 activity. (2) Since the functional interaction between $\mathrm{Bcl}-2$ and VDAC1 as well as maintaining a healthy balance between open and closed states of VDAC1 is crucial to maintain the normal function of mitochondria, we elected to avoid any downstream deleterious effects on VDAC1 gating that Bcl-2-like peptides may generate. Thus, to design SOD1-like peptides we first aimed at narrowing down the region in mutSOD1 that is responsible for binding to $\mathrm{Bcl}-2$. We used two complementary approaches. (1) Exon deletion approach: where we created a series of EGFP-SOD1-deletion mutants by deleting one exon at a time and then used these deletion mutants in co-IP experiments with Bcl-2 or to study their interaction with mitochondria in cells in situ. With this approach we generated five SOD1 (WT and G93A) constructs designated as full-length (FL) or delta $[(\Delta 1, \Delta 2$, $\Delta 3$, and $\Delta 5 ; \Delta 4$ was not created as this exon contains the G93A mutation)]. (2) Exon-generating approach: where we made small, exon-like SOD1 constructs only including one exon at the time (also tagged to EGFP), resulting in SOD1 A-D (without G93A mutation), $\mathrm{D}^{*}$ (with G93A mutation), or $\mathrm{E}$. As for the first approach, we used these exon-like SOD1 for co-IP experiments with $\mathrm{Bcl}-2$. Figure $4 A$ represents a map of SOD1 detailing each exon region (numeric and alphabetic). Using the exon-deletion approach we found that when we transfect these $\Delta$-SOD1s in HEK293T cells, while FL, $\Delta 3$, and $\Delta 5$ mutSOD1-G93A all cluster at the mitochondria in the presence of Bcl-2, $\Delta 2$ (and to a minor extent $\Delta 1$ ) does not (Fig. $4 B$ ). In fact, $\Delta 2$ mutSOD1-G93A presents a diffused staining across the cells similar to that of FL SOD1-WT (Fig. 4B). In co-IP, cell lysates were precipitated with the anti-Bcl-2 antibody. Western blot analysis of the precipitates using the anti-GFP antibody [ to visualize mutSOD1-G93A (FL or $\Delta)$ ] shows that while $\Delta 1, \Delta 3$, and $\Delta 5$ mutSOD1-G93A coprecipitate with Bcl-2 almost to the same extent as FL mutSOD1-G93A, $\Delta 2$ does not (Fig. $4 C$ ), indicating that the $\mathrm{N}$-terminal region encoded by exon 2 may be responsible for the binding with Bcl-2. Indeed, when we used the second approach and we transfected small exon-like SOD1 into the HEK293T cells and, again performed co-IPs with the anti-GFP antibody, we found that the two short exons ( $\mathrm{A}$ and $\mathrm{B}$ and mainly $\mathrm{AB}$ combined), corresponding to the $\mathrm{N}$-terminal region of mutSOD1, maintain the stronger binding affinity to Bcl-2 (Fig. 4D). Combined, these experiments indicate that SOD1 binds Bcl-2 in the region encoded by exon 2 and that the $\mathrm{N}$-terminal region may be the best to target for direct competition with mutSOD1 binding toward Bcl-2. We therefore designed five individual SOD1-like peptides (named P1-P5) corresponding to each of the five exons encoding SOD1 and tested whether they could inhibit the interaction between mutSOD1 and $\mathrm{Bcl}-2$. Of the five peptides, $\mathrm{P} 2$ is the most effective, inhibiting the binding between mutSOD1-G93A and Bcl-2 in HEK293T cells by $\sim 40 \%$ (Fig. $5 A$ ). $\mathrm{P} 2$ is also specific to the mutSOD1/Bcl-2 complex, as it does not affect the binding between mutSOD1-G93A and VDAC1 (Fig. 5B). Interestingly, P2 reduces clustering of mutSOD1G93A at the mitochondria in a concentration-dependent manner in HEK293T cells coexpressing FL mutSOD1-G93A and Bcl-2 (Fig. 5C). In these experiments, we cotransfected HEK293T cells with mutSOD1-G93A and Bcl-2 as in previous experiments and $20 \mathrm{~h}$ after transfection we treated the cells with increasing concentrations of P2 $(50,100$, and $200 \mathrm{ng})$ for $2 \mathrm{~h}$ and then analyzed the cells by confocal microscopy to follow mutSOD1-G93A localization and clustering at the mitochondria. Interestingly, at the higher dose (200 ng), treatment with P2 almost completely abolishes mitochondrial clustering of mutSOD1 and the largely cytosolic diffused pattern of mutSOD1-G93A strongly resembles that of $\Delta 2$ mutSOD1-G93A shown in Figure 4B. All together these data strongly indicate that $\mathrm{Bcl}-2$ mediates the interaction between misfolded mutSOD1 and the OMM.

\section{Small SOD1-like peptides against the mutSOD1/Bcl-2 complex prevent mitochondrial hyperpolarization and loss of viability in motor neuronal cells}

Since P2 is best able to diminish the interaction between mutSOD1-G93A and Bcl-2, we expect this peptide may have the greatest therapeutic value. We therefore began to fully characterize P2 in NSC-34 cells. We chose these cells for two main reasons. First, they are a motor neuronal cell line, and therefore share similarities with motor neurons (Cashman et al., 1992). Second, these cells have significant levels of endogenous Bcl-2. Thus, they can be used to determine the efficacy of the peptide(s) in destroying/preventing the binding between mutSOD1 and "native" Bcl-2 to eventually study its effect(s) on mitochondrial bioenergetics and cell viability.

To find out whether P2 is able to prevent the toxic binding between mutSOD1-G93A and endogenous Bcl-2, we first transduced the NSC-34 cells with adv-SOD1-G93A-EGFP for $72 \mathrm{~h}$ to ensure high and homogeneous expression of mutSOD1-G93A and then treated them with $50 \mathrm{ng}, 100 \mathrm{ng}$, or $200 \mathrm{ng}$ of $\mathrm{P} 2$ for $2 \mathrm{~h}$. At $74 \mathrm{~h}$, we harvested the cells and ran co-IPs to assess the binding. As seen in Figure 6A, P2 significantly diminishes the binding between mutSOD1 and Bcl-2 in a concentration-dependent manner. To further test the efficacy of $\mathrm{P} 2$, we performed a series of experiments to determine whether administration of $\mathrm{P} 2$ also functionally rescues mutSOD1/Bcl-2 caused damages. In cells in situ, mitochondrial membrane potential (MMP) can be measured by TMRE in a quantifiable manner if used at a low enough concentration (the nonquenching state of the dye) (Scaduto and Grotyohann, 1999). We therefore measured MMP in mutSOD1G93A NSC-34 cells with or without P2. Compared with SOD1WT, mutSOD1 hyperpolarizes mitochondria as evidenced by increased intensity in TMRE staining analyzed by confocal microscopy (Fig. 6C). TMRE-FACS analysis also confirmed that mutSOD1-G93A-transfected NSC-34 cells have hyperpolarized mitochondria (Fig. 6B). Treatment with P2 reverses the hyperpolarization, while treatment with a control scram- 
A

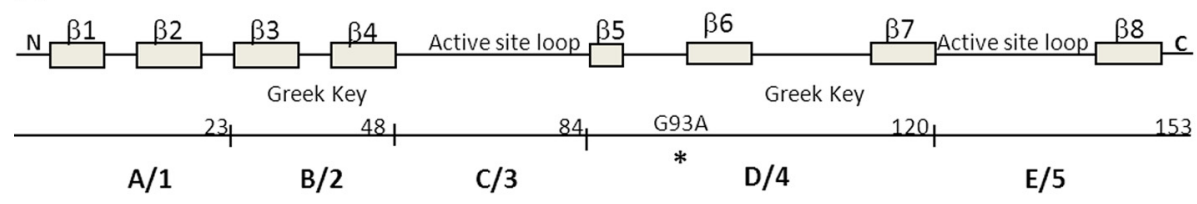

B
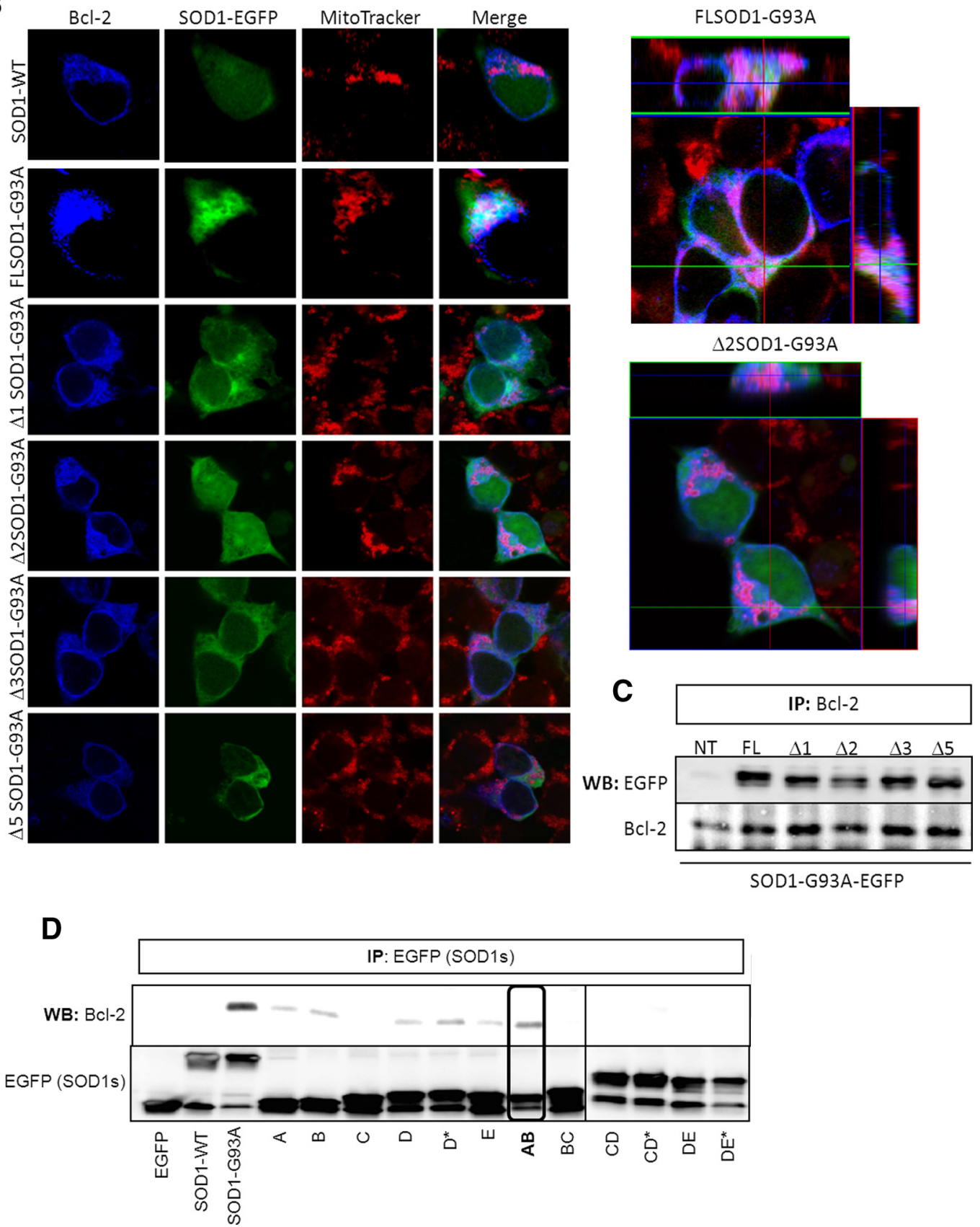

Figure 4. Exon 2 of mutSOD1 is responsible for binding with BCl-2. A, Illustration of the SOD1 exons. The G93A mutation is marked with *. B, C, HEK293T cells were cotransfected with FL or exon deletion S0D1-G93A constructs and BCl-2. Twenty-hours post-transfection, cells were either stained for BCl-2 and MitoTracker ( $\boldsymbol{B}$, with Z-stack on the right) or immunoprecipitated with anti-BCl-2 and probed for indicated antibodies (C). D, EGFP-tagged SOD1 (WT or G93A) with different exon domains expressed were cotransfected into HEK293T cells with BCl-2. The cells were harvested $24 \mathrm{~h}$ later and lysates immunoprecipitated with GFP antibody and probed with indicated antibodies.

bled peptide has no significant effect (Fig. $6 B, C$ ). Last, we performed a cell viability assay. SOD1 (WT or G93A)expressing NSC-34 cells were treated with different concentrations of P2 and a scramble P2 peptide as control. As for the binding experiments above, P2 significantly alleviates loss of viability in a concentration-dependent manner in mutSOD1G93A cells $(n=3, p<0.05)$, while the scramble P2 does not (Fig. 6D). 

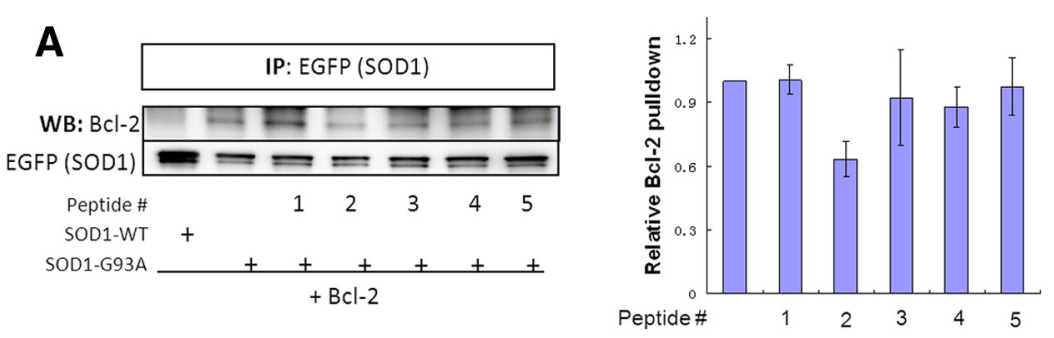

B

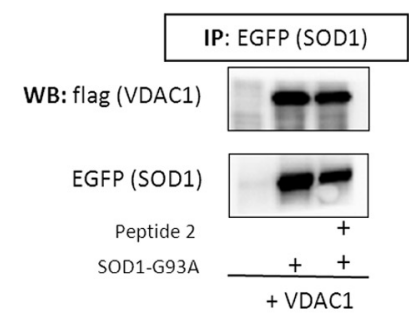

C

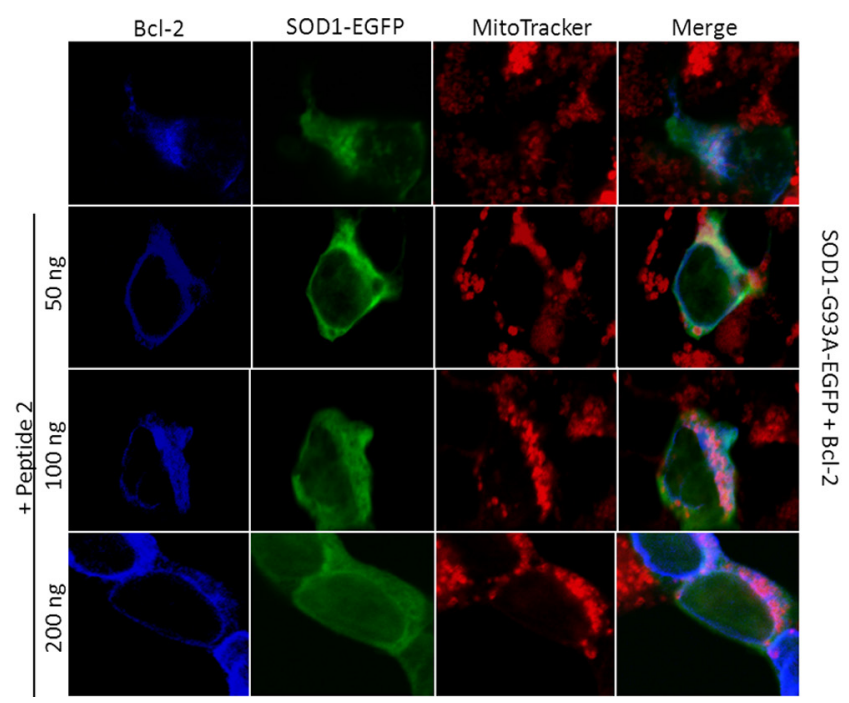

Figure 5. Peptide corresponding to exon 2-SOD1 inhibits mutSOD1/BCl-2 binding and resulting mutSOD1 mitochondrial clustering in HEK293T cells. A, Left, EGFP-tagged mutSOD1-G93A and BCl-2 were cotransfected into HEK293T cells. The lysates were aliquoted and mixed with $1 \mu \mathrm{m}$ peptide corresponding to each exon of SOD1. These mixtures were immunoprecipitated with GFP antibody and probed with antibodies indicated. Right, Quantifications of Western blots were normalized to the WT-SOD1 condition. B, EGFP-tagged mutSOD1-G93A and flag-tagged VDAC1 were cotransfected into HEK293T cells. The lysates were aliquoted and mixed with or without $1 \mu \mathrm{m}$ peptide 2 . These mixtures were immunoprecipitated with GFP antibody and probed with the antibodies indicated. C, HEK293T cells were cotransfected with mutSOD1-G93A and BCl-2. Twenty hours post-transfection, cells were treated with peptide $2(50,100$, or $200 \mathrm{ng})$, then stained with $300 \mathrm{~nm}$ MitoTracker. Mitochondrial localization and clustering of mutSOD1 were analyzed using confocal microscopy.

Small SOD1-like peptides against the mutSOD1/Bcl-2 complex restore ADP permeability in spinal cord mitochondria of symptomatic mutSOD1-G93A mice In an effort to test the therapeutic potential of SOD1-like peptides in disease, we first examined the ex-vivo effect of $\mathrm{P} 2$ on mitochondrial ADP permeability in spinal cord mitochondria isolated from the SOD1-G93A ALS mice. Figure 7A shows the purification profile of mitochondria isolated from the spinal cord of the mice at presymptomatic and symptomatic ALS disease stages. The mitochondrial fraction is enriched in mitochondrial proteins (Bcl-2, VDAC1, and cytochrome $c$ ), and is devoid of contamination from cytosol (actin) and endoplasmic reticulum (ER; calregulin). Compared with age-matched controls, mutSOD1G93A mitochondria of presymptomatic (Fig. 7C) and symptomatic (Fig. 7D) mice both consume ADP more slowly at low ADP concentration, consistent with our in vitro results in HEK293T cells (Fig. 3B,C) and previously published results (Mattiazzi et al., 2002, Israelson et al., 2010). Mathematical fitting (Lee et al., 1994) indicates $20-50 \%$ decrease of the OMM permeability to ADP in mitochondria isolated from mutSOD1-G93A mice compared with age-matched nontransgenic controls. All mitochondria show well coupled respiration following ADP addition even in the end stage, likely because the isolation procedure primarily yields relatively intact and healthy-looking mitochondria as examined by electron microscopy (Fig. 7B). However, the defect in ADP consumption indicates intrinsic defects in mitochondrial function and bioenergetics even in still morphologically intact mitochondria allowing for testing of the ability of the SOD1-like peptides to restore mitochondrial function before the final (and possibly not recoupable) mitochondrial disruption. We therefore isolated spinal cord mitochondria from symptomatic mutSOD1-G93A mice and incubated them with $\mathrm{P} 2$ before measuring ADP consumption. Compared with untreated mutSOD1-G93A mitochondria, P2 can fully restore mitochondrial ADP permeability in mutSOD1-G93A spinal cord to WT level (Fig. $7 E$ ) by decreasing the binding between mutSOD1 and Bcl-2 (data not shown). This strongly demonstrates that blocking the mutSOD1/Bcl-2 binding in disease can restore "normal" mitochondrial permeability, underscoring the need to target the initiating event in mutSOD1mediated toxicity and highlighting the therapeutic potentials of the SOD1-like peptides.

\section{Discussion}

Understanding the precise mechanisms by which mutSOD1 destroys multiple mitochondrial functions and identifying the mitochondrial targets of mutSOD1, is crucial to develop target-based therapies to rescue mitochondria in mutSOD1-ALS. We provide evidence that (1) the primary target of mutSOD1 at the mitochondria is $\mathrm{Bcl}-2$, (2) the initiating event in mutSOD1-induced mitochondrial dysfunction is the formation of the toxic mutSOD1/ Bcl-2 complex, and (3) preventing formation of the mutSOD1/Bcl-2 complex restores mitochondrial ADP permeability ex vivo in mitochondria of symptomatic mutSOD1G93A ALS mice as well as mitochondrial bioenergetics in situ in mutSOD1-expressing cells, ultimately preventing mutSOD1-induced cell loss. Thus, our data provide a solid rationale for the development of a targeted therapeutic approach that, by affecting the key steps leading to mitochondrial demise, may prove effective in broadly restoring mitochondrial functions, and eventually, preventing or delaying motor neuron death. 
Dissecting the precise mechanism(s) of toxicity of the mutSOD1/Bcl-2 complex, we identified VDAC1 as a downstream target of this complex. It has been proposed that mutant, but not WT, SOD1 targets VDAC1 directly. Misfolded mutSOD1 binds VDAC1 in spinal cord mitochondria and in vitro, in reconstituted lipid bilayer expressing only VDAC1 and mutSOD1 (thus lacking the necessary regulatory element of the OMM), it inhibits VDAC1 conductance (Israelson et al., 2010). Our data show that, rather than directly, mutSOD1 targets VDAC1 through the aberrant interaction with $\mathrm{Bcl}-2$. At the OMM, SOD1 (both WT and mutant) and VDAC1 independently bind and coprecipitate with Bcl-2. However, in situations in which the mutSOD1 accumulates at the mitochondria, mutSOD1 "sequesters" Bcl-2 triggering a conformational change that, ultimately, alters $\mathrm{Bcl}-2$ normal binding to VDAC1. Indeed, in the mutSOD1-G93A mice the nature of the binding between $\mathrm{Bcl}-2$ and VDAC1 changes over time becoming stronger as disease progresses. As Bcl-2 is targeted by the mutSOD1 during disease and gradually changes its conformation, the binding between $\mathrm{Bcl}-2$ and VDAC1 also changes: in diseased mutSOD1-G93A mice the anti-BH3-Bcl-2 antibody coprecipitates VDAC1 more efficiently that the anti-pocket-Bcl-2 antibody.

Functionally, Bcl-2 regulates VDAC1 gating (Arbel and Shoshan-Barmatz, 2010). In our model, and in the mutSOD1-G93A mice, formation of the mutSOD1/Bcl-2 complex not only alters the physical binding between VDAC1 and $\mathrm{Bcl}-2$, but also affects VDAC1 gating. In cells expressing both mutSOD1 and VDAC1, but low or no Bcl-2, VDAC1 conductance is not significantly reduced compared with control SOD1-WT cells, while it is reduced by $\sim 40 \%$ in cells where mutSOD1 is coexpressed with Bcl-2. Our data in HEK293T cells also indicate that the $\mathrm{BH} 3$ domain induces the partial closure of VDAC1 since inactivation of the $\mathrm{BH} 3$ domain (Bcl-2-AAA) and expression of the nontoxic mutSOD1/ Bcl-2-AAA completely restores ADP consumption. This fits with published data showing that $\mathrm{Bid}, \mathrm{a} \mathrm{BH} 3$ domain only protein, increases the probability of VDAC1 closure (Rostovtseva et al., 2004). In the mutSOD1-G93A mice, the correlation between the timing of formation of the mutSOD1/Bcl-2 complex (Pedrini et al., 2010), appearance of the exposed BH3 domain (Pedrini et al., 2010; Fig. 2), altered Bcl-2/VDAC1 binding (Fig. 2), and decreased ADP consumption (Fig. 7) also suggests that in vivo, it is the newly exposed $\mathrm{BH} 3$ domain that alters VDAC1 gating properties. Because these mutSOD1/Bcl-2-induced changes peak at disease onset but gradually develop over time in presymptomatic mice, we think they represent an early trigger of disease.

Based on our cell culture model we believe that, ultimately, the mitochondrial changes triggered by the mutSOD1/Bcl-2 complex lead to loss of metabolically active cells and, eventually, cell death.

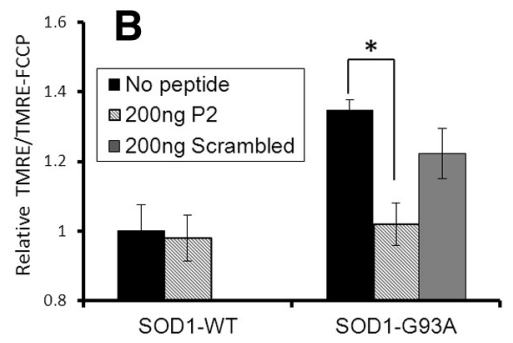

OD1-G93A-EGFP
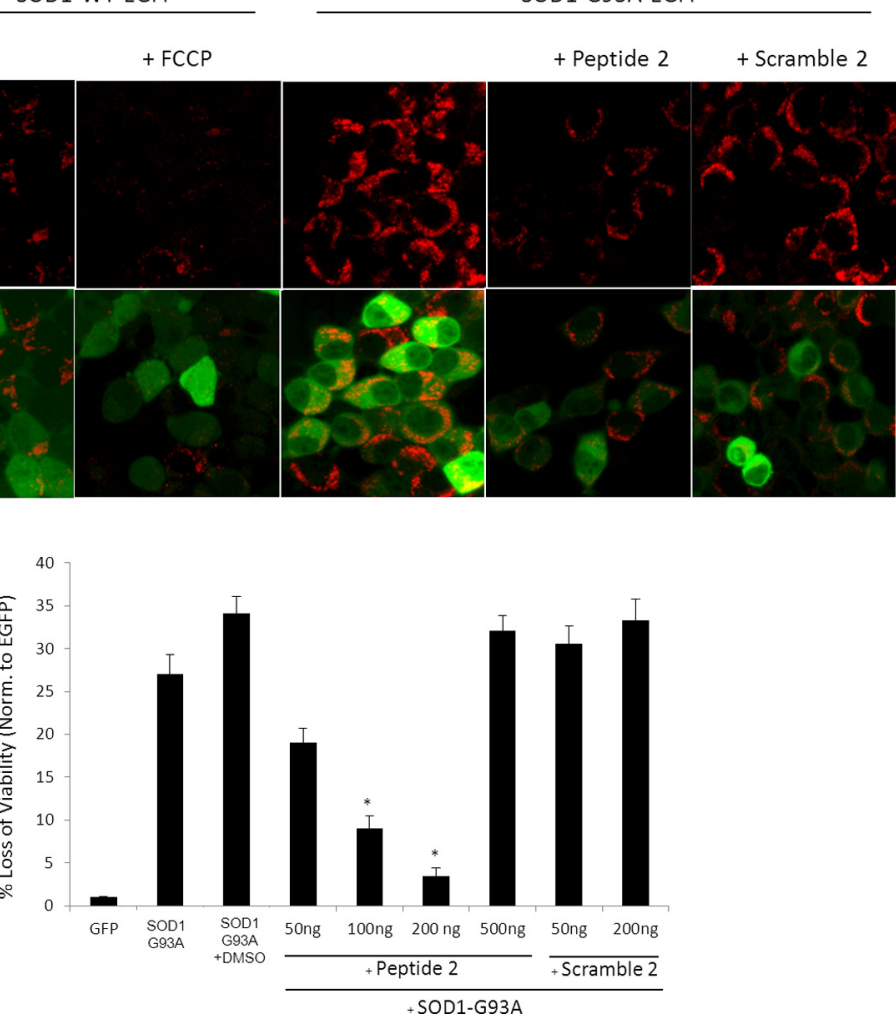

Figure 6. Peptide 2 reverses mutSOD1-caused hyperpolarization of MMP and loss of viability in NSC-34 cells. NSC-34 cells were transduced with adenovirus harboring SOD1 genes and treated with the indicated amount of peptides following protocols described (see with $25 \mathrm{~nm}$ TMRE in the presence or absence of $1 \mu \mathrm{m}$ FCCP and analyzed via flow cytometry $(\boldsymbol{B})$ or confocal microscopy (C). The ratio of TMRE and TMRE-FCCP staining was normalized to SOD1-WT treatment ( ${ }^{*} p<0.05, n=3$, compared with all other groups). $\boldsymbol{D}$, (ell viabilities were assessed with CellTiter-Glo and normalized to values of EGFP control $\left({ }^{*} p<0.05, n=3\right)$.

Again, the key mitochondrial mediator of the mutSOD1 toxicity is Bcl-2, which partially works through VDAC1 but also affects other mitochondrial pathways in parallel. In fact, inactivation of the toxic $\mathrm{BH} 3$ domain in Bcl-2 equally abolishes toxicity of the mutSOD1/ $\mathrm{Bcl}-2$ complex regardless of VDAC1 (mutSOD1/Bcl-2-AAA is no longer toxic in both VDAC1-positive and knock-down cells). Knocking down VDCA1, at least to $80 \%$ of normal levels, rescues the cells against the mutSOD1/Bcl-2 toxicity confirming that VDAC1 acts downstream of the mutSOD1/Bcl-2 complex to regulate ADP permeability.

Ultimately, whether mutSOD1 damages mitochondria by directly inhibiting VDAC1 or indirectly through the conformationally changed $\mathrm{Bcl}-2$, and whether the mutSOD1/Bcl-2 complex primarily acts via VDAC1 or also alters mitochondria through additional mechanisms (e.g., OMM hyperpolarization) has major implications on how we design therapeutics. Additionally, since VDAC does not simply act as a "door" that can be opened or closed (Colombini, 2012), but rather functions through dynamic 
A
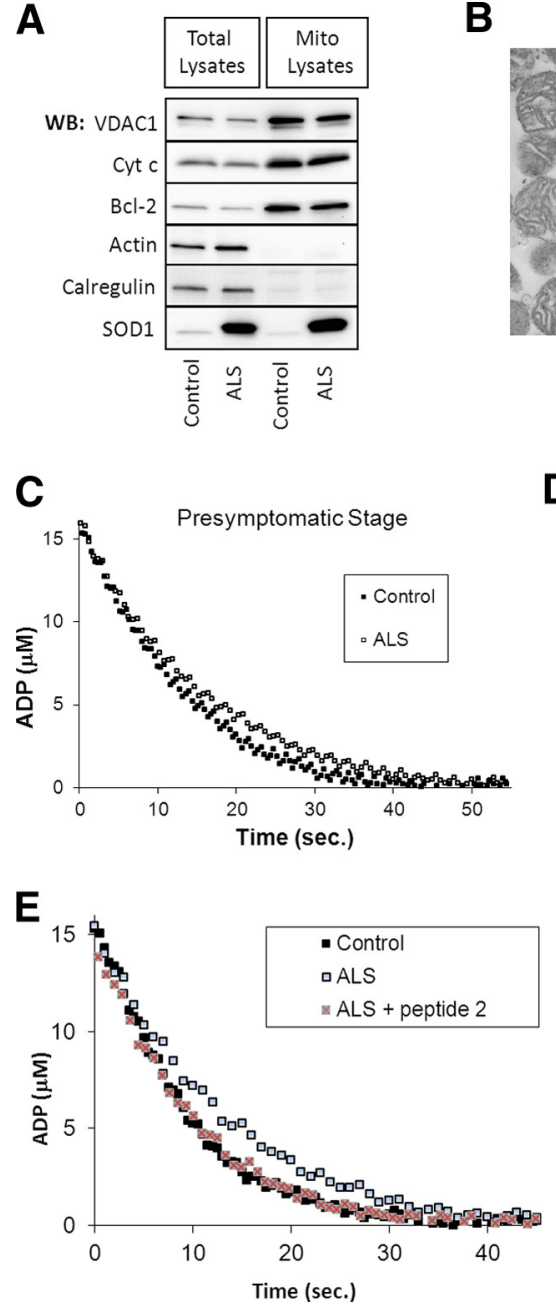

B

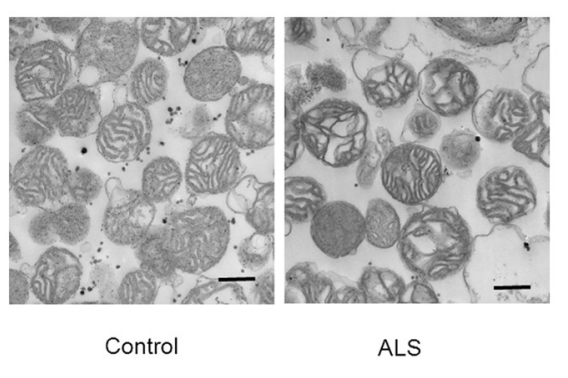

D

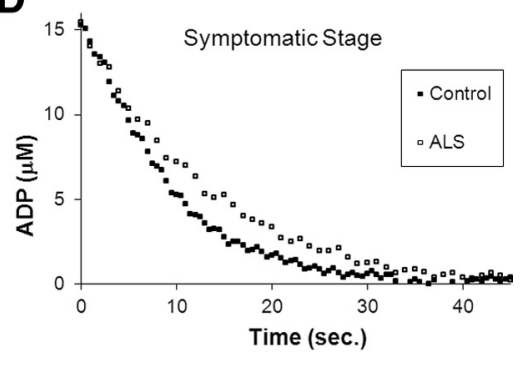

ing as to how to intervene to maintain its homeostasis toward one state versus the other.

Indeed, here we show two sets of results that suggest that in the context of mutSOD1-ALS, the state (open or close) of VDAC1 is unclear and that VDAC1 is not the primary mediator of mutSOD1 toxicity at the mitochondria. First, we show that in mutSOD1 mitochondria (from mutSOD1 cells and mice) ADP consumption rate is reduced consistent with VDAC1 closure and that this VDAC1 closure is downstream of the mutSOD1/ $\mathrm{Bcl}-2$ complex, which is no longer toxic in VDAC1 knockdown cells. However, at a closer analysis, the result in VDAC1 knockdown cells raises a puzzling question. If VDAC1 closure is a key element of mutSOD1 toxicity that supposedly drives disease, how would eliminating VDAC1 (and therefore closing it completely) rescue against mutSOD1 toxicity? Second, we find hyperpolarized mitochondria in mutSOD1/Bcl-2 cells: a finding that does not match with a permanent VDAC1 closure, which would predict depolarization of OMM due to ineffective entry of metabolites into the mitochondria. Instead, we find hyperpolarized mitochondria; thus it is likely that the mutSOD1/Bcl-2 complex affects the mitochondria through two parallel pathways and that it induces hyperpolarization not through partial VDAC1 closure but directly by overwhelming the mitochondria with excessive reactive oxygen species generation. It would explain why genetic deletion of VDACl in the mutSOD1-G37R mice accelerates disease onset but does not affect disease progression and, overall, does not have a large impact on survival (Israelson et al., 2010).

Figure 7. Peptide 2 rescues mutSOD1/BCl-2-induced inhibition of mitochondrial ADP permeability. $\boldsymbol{A}$, Western blot (WB) analysis of the isolated mitochondrial lysate (Mito Lysate) and spinal cord lysate (Total Lysate). $\boldsymbol{B}$, Comparison of the morphology of mitochondria isolated from symptomatic stage and its age-matched control under transmission electron microscopy. Scale bar, $500 \mathrm{~nm} . \mathbf{C}, \mathbf{D}, \mathrm{ADP}$ consumption curve of isolated spinal cord mitochondria through disease courses (black dots): (C) presymptomatic, (D) symptomatic, and their age-matched controls (empty dots). $\boldsymbol{E}$, ADP consumption curve of isolated spinal cord mitochondria from symptomatic mutSOD1-G93A mice with or without treatment of $1 \mu \mathrm{m}$ peptide 2.

gating properties; targeting VDAC therapeutically through drugs that either keep it in a constant open state (as suggested by the lipid bilayer observation that mutSOD1 closes VDAC1) or close it completely may both be deleterious to the mitochondria. VDAC is the major throughway for ions, metabolites, and nucleotides from the mitochondria to the cytosol (Colombini, 2012). In the open state, VDAC shows preference for anions conducting metabolites such as ATP and succinate (Rostovtseva and Colombini, 1997). In the closed state, VDAC works as a smaller channel with preference to cations. Closing of VDAC results in a strong barrier to anions like ATP, which becomes nonpermeant (Rostovtseva and Colombini, 1997). The reverse happens for cations, mainly $\mathrm{Ca}^{2+}$; when VDAC is excessively open $\mathrm{Ca}^{2+}$ does not shuttle properly from the cytosol to the mitochondria (Tan and Colombini, 2007). Most of what we know about VDAC gating comes from studies in reconstituted lipid bilayer, a very useful but highly artificial system where at any given time VDAC can be opened or closed. In the milieu of the OMM, teasing out the actual state of such a dynamic channel, mainly in the context of a progressive disease, is extremely challenging and can be mislead-
Regardless of whether mutSOD1/Bcl-2 affects mitochondrial function through one or more mechanisms, our data show that this mutSOD1/Bcl-2 complex is the upstream toxic event and suggest that, therapeutically, the mutSOD1/Bcl-2 complex is the primary target to modify in the effort to rescue mitochondrial and cell function in mutSOD1-mediated ALS. Our approach to design small SOD1-like peptides that mimic the binding region of SOD1 to Bcl-2 has yielded a promising peptide (P2) of 32 aa corresponding to the $\mathrm{N}$-terminal region of SOD1 encoded by exon 2. P2 abolishes the binding between mutSOD1 and Bcl-2 in a dose-dependent manner and prevents clustering of mutSOD1 at the mitochondria, confirming that $\mathrm{Bcl}-2$ drives the interaction of mutSOD1 with the OMM. Not only does P2 block formation of the mutSOD1/Bcl-2 complex and prevent the physical interaction between mutSOD1 and the mitochondria, it also restores mitochondrial ADP permeability in spinal cord mitochondria isolated from symptomatic mutSOD1-G93A mice, prevents mitochondria hyperpolarization in motor neuron-like cells expressing mutSOD1, and reduces cell death. It is important to note that in cases where the aberrant binding between mutSOD1 and 
BCl-2 already occurred, $\mathrm{P} 2$ is able to reverse its toxic effects: it restores bioenergetics in mitochondria isolated from symptomatic mice and in cells with already hyperpolarized mitochondria.

The efficacy of the P2 peptide in rescuing different aspects of mutSOD1 toxicity on mitochondria (and their downstream effects on cell survival) confirms that Bcl-2 is a primary mediator of mutSOD1 toxicity and that formation of the toxic mutSOD1/ Bcl-2 complex is a key step in the series of events that lead to mitochondrial dysfunction. Importantly, our data show the therapeutic promise of the $\mathrm{P} 2$ peptide, which is not toxic in cells and is effective in restoring ADP permeability in ex vivo mitochondria of symptomatic mice without directly changing the status of VDAC1 (thus avoiding any undesired secondary effects on VDAC1).

Whether this peptide approach against mutSOD1/Bcl-2 has therapeutic implications beyond the limited number of mutSOD1 patients is not known. Our recent finding that a subset $(\sim 30 \%)$ of sporadic ALS patients has an over-oxidized SOD1-WT with features similar to mutSOD1 including aberrant binding to Bcl-2 and mitochondrial dysfunction (Guareschi et al., 2012) offers hope to extend the approach to a selected non-mutSOD1 group of patients.

\section{References}

Arbel N, Shoshan-Barmatz V (2010) Voltage-dependent anion channel 1-based peptides interact with $\mathrm{Bcl}-2$ to prevent antiapoptotic activity. J Biol Chem 285:6053-6062. CrossRef Medline

Cashman NR, Durham HD, Blusztajn JK, Oda K, Tabira T, Shaw IT, Dahrouge S, Antel JP (1992) Neuroblastoma $\times$ spinal cord (NSC) hybrid cell lines resemble developing motor neurons. Dev Dyn 194:209-221. CrossRef Medline

Cheng EH, Kirsch DG, Clem RJ, Ravi R, Kastan MB, Bedi A, Ueno K, Hardwick JM (1997) Conversion of Bcl-2 to a Bax-like death effector by caspases. Science 278:1966-1968. CrossRef Medline

Colombini M (2012) VDAC structure, selectivity, and dynamics. Biochim Biophys Acta 1818:1457-1465. CrossRef Medline

Damiano M, Starkov AA, Petri S, Kipiani K, Kiaei M, Mattiazzi M, Flint Beal M, Manfredi G (2006) Neural mitochondrial Ca2 + capacity impairment precedes the onset of motor symptoms in $\mathrm{G} 93 \mathrm{~A} \mathrm{Cu} / \mathrm{Zn}$-superoxide dismutase mutant mice. J Neurochem 96:1349-1361. CrossRef Medline

Guareschi S, Cova E, Cereda C, Ceroni M, Donetti E, Bosco DA, Trotti D, Pasinelli P (2012) An over-oxidized form of superoxide dismutase found in sporadic amyotrophic lateral sclerosis with bulbar onset shares a toxic mechanism with mutant SOD1. Proc Natl Acad Sci U S A 109:50745079. CrossRef Medline

Higgins CM, Jung C, Ding H, Xu Z (2002) Mutant Cu, Zn superoxide dismutase that causes motoneuron degeneration is present in mitochondria in the CNS. J Neurosci 22:RC215. Medline

Israelson A, Arbel N, Da Cruz S, Ilieva H, Yamanaka K, Shoshan-Barmatz V, Cleveland DW (2010) Misfolded mutant SOD1 directly inhibits VDAC1 conductance in a mouse model of inherited ALS. Neuron 67:575587. CrossRef Medline

Jung C, Higgins CM, Xu Z (2002) Mitochondrial electron transport chain complex dysfunction in a transgenic mouse model for amyotrophic lateral sclerosis. J Neurochem 83:535-545. CrossRef Medline

Kirkinezos IG, Bacman SR, Hernandez D, Oca-Cossio J, Arias LJ, PerezPinzon MA, Bradley WG, Moraes CT (2005) Cytochrome c association with the inner mitochondrial membrane is impaired in the CNS of G93ASOD1 mice. J Neurosci 25:164-172. CrossRef Medline

Kong J, Xu Z (1998) Massive mitochondrial degeneration in motor neurons triggers the onset of amyotrophic lateral sclerosis in mice expressing a mutant SOD1. J Neurosci 18:3241-3250. Medline

Kristián T, Hopkins IB, McKenna MC, Fiskum G (2006) Isolation of mitochondria with high respiratory control from primary cultures of neurons and astrocytes using nitrogen cavitation. J Neurosci Methods 152:136-143. CrossRef Medline

Lee AC, Zizi M, Colombini M (1994) Beta-NADH decreases the permeability of the mitochondrial outer membrane to ADP by a factor of 6 . J Biol Chem 269:30974-30980. Medline

Li Q, Vande Velde C, Israelson A, Xie J, Bailey AO, Dong MQ, Chun SJ, Roy T, Winer L, Yates JR, Capaldi RA, Cleveland DW, Miller TM (2010) ALS-linked mutant superoxide dismutase 1 (SOD1) alters mitochondrial protein composition and decreases protein import. Proc Natl Acad Sci U S A 107:21146-21151. CrossRef Medline

Liu J, Lillo C, Jonsson PA, Vande Velde C, Ward CM, Miller TM, Subramaniam JR, Rothstein JD, Marklund S, Andersen PM, Brännström T, Gredal O, Wong PC, Williams DS, Cleveland DW (2004) Toxicity of familial ALS-linked SOD1 mutants from selective recruitment to spinal mitochondria. Neuron 43:5-17. CrossRef Medline

Mattiazzi M, D’Aurelio M, Gajewski CD, Martushova K, Kiaei M, Beal MF, Manfredi G (2002) Mutated human SOD1 causes dysfunction of oxidative phosphorylation in mitochondria of transgenic mice. J Biol Chem 277:29626-29633. CrossRef Medline

Pallotti F, Lenaz G (2007) Isolation and subfractionation of mitochondria from animal cells and tissue culture lines. Methods Cell Biol 80:3-44. CrossRef Medline

Parsons DF, Williams GR, Chance B (1966) Characteristics of isolated and purified preparations of the outer and inner membranes of mitochondria. Ann NY Acad Sci 137:643-666. CrossRef Medline

Pasinelli P, Brown RH (2006) Molecular biology of amyotrophic lateral sclerosis: insights from genetics. Nat Rev Neurosci 7:710-723. CrossRef Medline

Pasinelli P, Belford ME, Lennon N, Bacskai BJ, Hyman BT, Trotti D, Brown RH Jr (2004) Amyotrophic lateral sclerosis-associated SOD1 mutant proteins bind and aggregate with Bcl-2 in spinal cord mitochondria. Neuron 43:19-30. CrossRef Medline

Pedrini S, Sau D, Guareschi S, Bogush M, Brown RH Jr, Naniche N, Kia A, Trotti D, Pasinelli P (2010) ALS-linked mutant SOD1 damages mitochondria by promoting conformational changes in Bcl-2. Hum Mol Genet 19:2974-2986. CrossRef Medline

Rosen DR, Siddique T, Patterson D, Figlewicz DA, Sapp P, Hentati A, Donaldson D, Goto J, O'Regan JP, Deng HX (1993) Mutations in Cu/Zn superoxide dismutase gene are associated with familial amyotrophic lateral sclerosis. Nature 362:59-62. CrossRef Medline

Rostovtseva T, Colombini M (1997) VDAC channels mediate and gate the flow of ATP: implications for the regulation of mitochondrial function. Biophys J 72:1954-1962. CrossRef Medline

Rostovtseva TK, Antonsson B, Suzuki M, Youle RJ, Colombini M, Bezrukov SM (2004) Bid, but not Bax, regulates VDAC channels. J Biol Chem 279:13575-13583. CrossRef Medline

Scaduto RC Jr, Grotyohann LW (1999) Measurement of mitochondrial membrane potential using fluorescent rhodamine derivatives. Biophys J 76:469-477. CrossRef Medline

Schimmer AD, Hedley DW, Penn LZ, Minden MD (2001) Receptor- and mitochondrial-mediated apoptosis in acute leukemia: a translational view. Blood 98:3541-3553. CrossRef Medline

Sims NR, Anderson MF (2008) Isolation of mitochondria from rat brain using Percoll density gradient centrifugation. Nat Protoc 3:1228-1239. CrossRef Medline

Szasz G, Gruber W, Bernt E (1976) Creatine kinase in serum: 1. Determination of optimum reaction conditions. Clin Chem 22:650-656. Medline

Tan W, Colombini M (2007) VDAC closure increases calcium ion flux. Biochim Biophys Acta 1768:2510-2515. CrossRef Medline

Tan W, Loke YH, Stein CA, Miller P, Colombini M (2007) Phosphorothioate oligonucleotides block the VDAC channel. Biophys J 93:1184-1191. CrossRef Medline

Vande Velde C, Miller TM, Cashman NR, Cleveland DW (2008) Selective association of misfolded ALS-linked mutant SOD1 with the cytoplasmic face of mitochondria. Proc Natl Acad Sci U S A 105:4022-4027. CrossRef Medline 